\title{
Subspace Subcodes of Reed-Solomon Codes ${ }^{1}$
}

\author{
Masayuki Hattori Robert J. McEliece Wei Lin \\ California Institute of Technology \\ Pasadena, California 91125, USA
}

\begin{abstract}
A subspace subcode of a Reed-Solomon (SSRS) code over $G F\left(2^{m}\right)$ is the set of RS codowords, whose components all lie in a particular $G F(2)-$ subspace of $G F\left(2^{m}\right)$. SSRS codes include both generalized BCH codes and "trace-shortened" RS codes [2][3] as special cases. In this paper we present an explicit formula for the dimension of an arbitrary RS subspace subcode. Using this formula, we find that in many cases, SSRS codes are competetive with algebraic geometry codes, and that in some cases, the dimension of the best subspace subcode is larger than that of the corresponding GBCH code.
\end{abstract}

I. Introduction. Definition of SSRS Codes. Let $\mathcal{C}$ be the $\left(n, k_{0}, d_{0}\right) \mathrm{RS}$ code over $G F\left(2^{m}\right)$ defined by the parity-check polynomial $h(x)=\prod_{j=1}^{k_{0}}\left(x-\alpha^{j}\right)$, where $\alpha$ is a primitive $n$th root of unity in $G F\left(2^{m}\right)$, and $d_{0}=n-k_{0}+1$. Let $\mathcal{S}$ be a $\nu$-dimensional $G F(2)$-subspace of $G F\left(2^{m}\right)$. We define the subspace subcode $\mathcal{C}_{S}$ to be the set of all codewords from $\mathcal{C}$, whose components all lie in $\mathcal{S}$. The code $\mathcal{C}_{S}$ is thus 8 cyclic group code of length $n$ over the Abelien group $S$ of size $2^{\nu}$, with minimum distance at least $\alpha_{0}$. For example, if $S=G F\left(2^{\nu}\right)$, where $\nu / m$, the corresponding SSRS code is a generalized BCH cade. Also, if we consider subspaces $\mathcal{S}$ which have dual bases of the form $\left\{1, \alpha, \alpha^{2}, \ldots, \alpha^{\mu-1}\right\}$, we obtain the class of "TSRS" codes [3], which are in turn a generalization of the codes introduced by Solomon [2].

II. Main Result. Dimension Formula.

Suppose $\mathcal{S}$ has basis $\left\{\beta_{0}, \ldots, \beta_{\nu-1}\right\}$ and "trace-dual" basis $\left\{\gamma_{0}, \ldots, \gamma_{\mu-1}\right\}$, where $\mu=m-\nu$ and $\operatorname{Tr}_{i}^{m}\left(\beta_{i} \gamma_{j}\right)=0$ for all $i, j$. Let $\left\{j 2^{i}: i=0 \ldots, d_{j}-1\right\}$ be the $j$ th cyclotomic coset modulo $n$, where $d_{j}$ is the degree of $j \bmod n$. Let $J=\left\{1,2, \ldots, k_{0}\right\}$. We define the $j$ th index set $A_{j}$ for the SSRS code $\mathcal{C}_{S}$ as $A_{j}=$ $\left\{i: 0<i<m-1\right.$ and $\left.j \cdot 2^{i} \bmod n \in J\right\}$, with $\left|A_{j}\right|=a_{j}$. We define the $j$ th cyclotomic matrix for $\mathcal{C}_{s}$ to be the $\mu \times a_{j}$ matrix $\Gamma_{j}$ whose $(h, i)$ th entry is $\gamma_{h}^{2^{i}}$ where $h=0, \ldots, \mu-1$ and $i \in A_{j}$. The rank of $\Gamma_{j}$ is denoted by $r_{j}$.

Theorem 1. The binary dimension of the SSRS code as dofined above is given by the formula

$$
K(\mathcal{C}, S)=\sum_{j \in I_{n}} d_{j}\left(a_{j}-r_{j}\right),
$$

where $I_{n}$ denotes a complete set of representatives of the modulo $n$ cyclotomic cosets. Thus $\mathcal{C}_{S}$ is an $\left(n, k, d_{0}^{+}\right)$cyclic group code over $\mathcal{S}$, where $k=K(\mathcal{C}, \mathcal{S}) / \nu$. (The notation d $d_{0}^{+}$means that the true minimum distance of the code is at least $d_{0}$.)

'Wei Lin's contribution, and a portion of Robert McEliece's contribution, was supported by AFOSR grant no. F49620-94-1-005. A portion of MaEliece's contribution was done at the Jet Proput sion Laboratory, Californis Institute of Technology, under contract to the National Aeronsutica and Space Administration. Masayuki Hattori's contribution was aponsored by the Sony Corporation.
Since $\Gamma_{j}$ is a $\mu \times a_{j}$ matrix, it follows that $r_{j} \leq \min \left(\mu, a_{j}\right)$, and so we have the following Corollary.

Corollary 1. With the same hypotheses as Theorem 1, we have the lower bound

$$
K(\mathcal{C}, \mathcal{S}) \geq \sum_{j \in I_{n}} \max \left(d_{j}\left(a_{j}-\mu\right), 0\right) .
$$

It is possible to show that if $\mathcal{S}$ has a dual basis of the form $\left\{1, \beta, \ldots, \beta^{\mu-1}\right\}$, where $\beta$ is an element of degree $m$ in $G F\left(2^{m}\right)$, then the lower bound of Corollary 1 is exact, for all parent RS codes $\mathcal{C}$. (This result is a small generalization of the main result proved in [3].) In fact, extensive numerical experimentation indicates that "most" subspaces $\mathcal{S}$ have this property, although we do not have a fully satisfactory theoretical explanation of this phenomenon.

\section{EXAMPLES AND CONCLUSION.}

Example 1. Let $m=4, n=15, k_{0}=9$ and $\nu=2$. Suppose $S_{0}$ and $S_{1}$ are spanned by $\{1, \alpha\}$ and $\left\{1, \alpha^{5}\right\}$, respectively. Starting from a parent $(15,9,7)$ RS code $\mathcal{C}$, and using Theorem 1 , we find that $\mathcal{C}_{\mathcal{S}_{0}}$ is a $\left(15,6,7^{+}\right)$code over $\mathcal{S}_{0}$ and $\mathcal{C}_{S_{1}}$ is a $\left(15,7,7^{+}\right)$code over $S_{1} . \mathcal{C}_{S_{0}}$ is equivalent to a TSRS code given in [3]. On the other hand, since $\mathcal{S}_{1}=G F(4), \mathcal{C}_{S_{1}}$ is a GBCH code.

Example 2. Let $m=6, n=63, k_{0}=53$ and $\nu=2$. Suppose $S_{0}$ and $S_{1}$ are spanned by $\left\{1, \alpha^{0}\right\}$ and $\left\{1, \alpha^{21}\right\}$, respectively. (Thus $S_{1}=G F(4)$.) Starting from a parent $(63,53,11)$ RS code, and using Theorem 1 , we find that $\mathcal{C}_{S_{0}}$ is a $\left(63,42.5,11^{+}\right)$ code over $\mathcal{S}_{0}$, and $\mathcal{C}_{\mathcal{S}_{1}}$ is a $\left(63,41,11^{+}\right)$GBCH code over $G F(4)$. Note that the dimension of $\mathcal{C}_{S_{0}}$ is higher than that of the GBCH code $\mathcal{C}_{S_{1}}$.

The only codes with parameters comparable to subspace subcodes that we are aware of are the algebraic geometry (AG) codes, e.g., [1]. For a given length $n$ and alphabet size $q$, highrate subspace subcodes are often superior to AG codes. Furthermore, decoding a subspace subcode up to the designed distance $d_{0}$ is almost the same as for the parent RS codes, which of course is quite easy. Thus subspace subcodes may provide an attractive alternative to AG codes in certain practical applications, such as outer codes in concatenated coding schemes.

\section{REFERENCES}

[1] A. M. Barg G. L. Katsman and M. A. Tsfasman, "AlgebraicGeometric Codes from Curves of Small Genus." Problemy Peredachi Informatsii, Vol. 23, No. 1, (January-March 1987) pp. 42-46.

[2] Gustave Solomon, "Nonlinear, Nonbinary Cyclic Group Codea." JPL TDA Progres Report vol. 43-108 (February 1992), pp. 8495. See also Proc. ISIT 93, p. 192.

[3] R. J. McEliece and G. Solomon, "Trace-Shartened ReedSolomon Codes." Proc. 2nd International Symposium on Communication Theory and Applications, Ambleside, UK, July 1993. 